\title{
Evidence for "Preterminal" Nicotinic Receptors on GABAergic Axons in the Rat Interpeduncular Nucleus
}

\author{
Clément Léna, Jean-Pierre Changeux, and Christophe Mulle \\ URA CNRS D1284, Laboratoire de Neurobiologie Moléculaire, Département des Biotechnologies, Institut Pasteur, 75724 \\ Paris Cedex 15, France
}

\begin{abstract}
Presynaptic nicotinic ACh receptors (nAChRs) are abundant in the nervous system, where they are thought to regulate the release of various neurotransmitters. Whole-cell recordings performed on rat interpeduncular nucleus neurons using the thin-slice technique showed that nicotine dramatically increased the frequency of postsynaptic GABAergic currents. This effect was observed at low micromolar concentration of agonist; it was mimicked by cytisine, dimethylphenylpiperazinium, and $\mathrm{ACh}$ in the presence of eserine. It was blocked by hexamethonium, dihydro- $\beta$-erythroidine, and mecamylamine. The presynaptic action was suppressed in the presence of JTX. A comparable effect of nicotine was found using a preparation of acutely isolated neurons that had retained synaptic terminals attached to their cell body as evidenced by immunoreactivity to synaptophysin and presence of spontaneous GABAergic and glutamatergic synaptic activity. Nicotinic agonists increased the frequency of GABAergic postsynaptic currents, an effect blocked by curare and mecamylamine. This action was also suppressed in the presence of TTX. These data suggest the presence of nAChRs at a preterminal level on axons of intrinsic GABAergic neurons. We propose that, in contrast to presynaptic nAChRs, activation of these "preterminal" nAChRs can trigger a spike discharge and thus have a generalized action on the GABAergic afferent.
\end{abstract}

[Key words: neuronal nicotinic receptor, interpeduncular nucleus, presynaptic regulation, preterminal receptors, GABAergic synaptic currents, thin-slice patch-clamp techniques]

The presence of a large variety of nicotinic $\mathrm{ACh}$ receptors (nAChRs) in the mammalian CNS has been recently demonstrated at a structural and functional level (see Sargent, 1993, for review). Various combinations of genes encoding $\alpha$ - and $\beta$-subunits of the nAChR family are cxpressed in distinct regions of the CNS. The functional properties of several $n A C h R$ sub-

\footnotetext{
Received Aug. 12, 1992; revised Jan. 11, 1993; accepted Jan. 13, 1993.

We thank Antoine Triller for his help with the immunocytochemistry and for the micrographs. We thank Pierre Vincent for the computer program for PSC analysis. We also thank Ottavio Arancio, Pierse Benoit, and Richard Miles for helpful discussion throughout this work. This work was supported by grants from the Association Francaise contra la Myopathie, the College de France, the Centre National de la Recherche Scientifique, the Ministere de la Recherche, and the Direction des Recherches, Etudes et Techniques (Contract 87/211).

Correspondence should be addressed to Christophe Mulle, Molecular Neurobiology Laboratory, The Salk Institute, 10010 North Torrey Pines Boulevard, La Jolla, CA 92037.

Copyright @ 1993 Society for Neuroscience 0270-6474/93/132680-09\$05.00/0
}

types have been described in different CNS regions (Lipton et al., 1987; Mulle and Changeux, 1990; Alkondon and Albuquerque, 1991; Mulle et al., 1991). These subtypes not only are distinct from peripheral nAChRs but also differ from one another in their pharmacological and electrophysiological properties. Furthermore, nAChRs can be differentially located on the surface of the neuron. Evidence exists for the presence of postsynaptic nAChRs located on the somatodendritic membrane [in the substantia nigra pars compacta, the ventral tegmental area, the medial habenula, the interpeduncular nucleus (IPN), and retinal ganglion cells; see Sargent, 1993, for review], where their activation leads to membrane depolarization and triggering of action potentials. On the other hand, a large number of anatomical and biochemical studies point to an abundance of presynaptic nAChRs located at the level of axon terminals.

Lesion experiments combined with ligand binding studies or immunocytochemical methods have demonstrated axonal transport of $\mathrm{nAChRs}$ in the fasciculus retroflexus (Clarke et al., 1986), retinotectal fibers (Swanson et al., 1987), or thalamocortical fibers in the cat (Prusky et al., 1987) and the rat (Sahin et al., 1992). Most studies on presynaptic nAChRs have involved measurement of the release of various neurotransmitters either in brain slices or in synaptosomes (see Wonnacott et al., 1990, for review). For instance, in ascending dopaminergic systems, nicotinic agonists facilitate dopamine release from striatal nerve terminals both in vivo (Giorguieff et al., 1976) and in vitro (Westfall, 1974, slice; Rapier et al., 1988, synaptosomes). Nicotine also regulates release of neurotransmitters such as $\mathrm{ACh}$, noradrenalin, 5-HT, and GABA by acting at receptors located at nerve terminals located in various brain regions (sce Wonnacott et al., 1990, for review).

There have been few attempts to clarify the role of these receptors by electrophysiological means. Nicotinic agonists decrease the amplitude of the field potential evoked by stimulation of the fasciculus retroflexus (Brown et al., 1983; Mulle et al., 1991 ) or the goldfish optic nerve (King, 1990). In rat neocortex, nicotine increases the amplitude of glutamatergic EPSPs (Vidal and Changeux, 1993), possibly by activation of presynaptic receptors. Electrophysiological studies have also shown that, at the neuromuscular junction, $\mathrm{ACh}$ facilitates its own release by activation of presynaptic receptors (see Wessler, 1992, for review).

There is some agreement about the putative mode of action of presynaptic receptors. Studies utilizing brain slices in the presence of TTX or isolated nerve terminals (see Wonnacott et al., 1990, for review) have indicated that nicotine can act directly on the presynaptic terminal to increase transmitter release 
A1

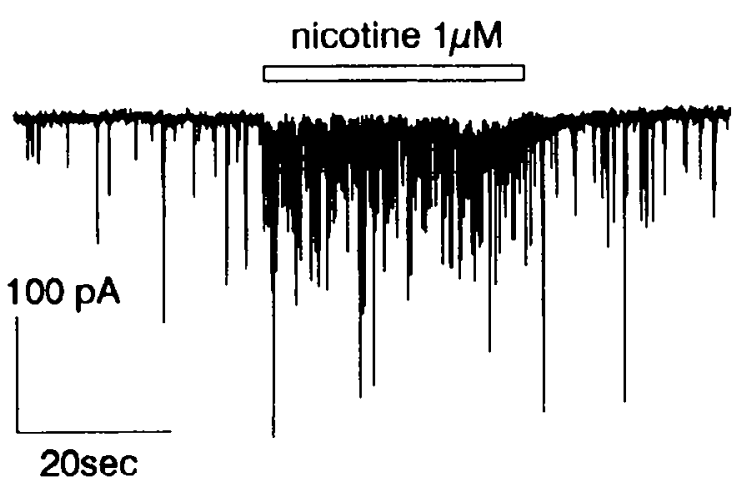

A2

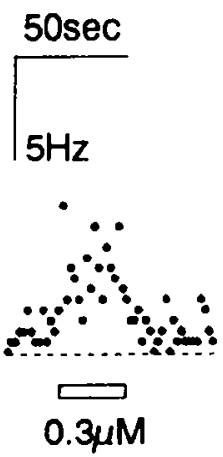

B1

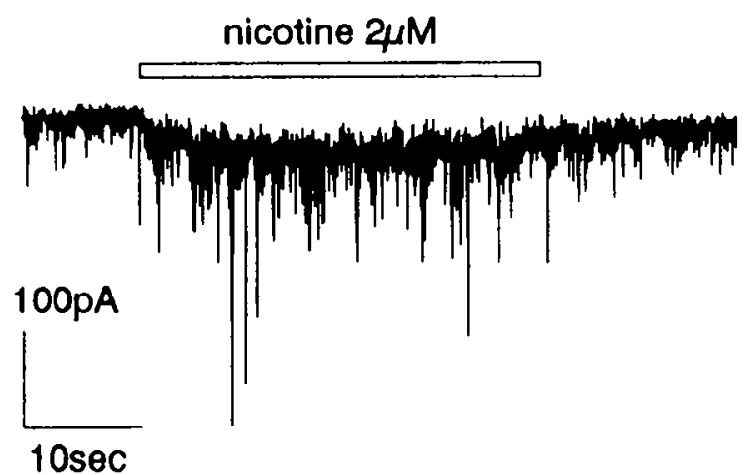

B2

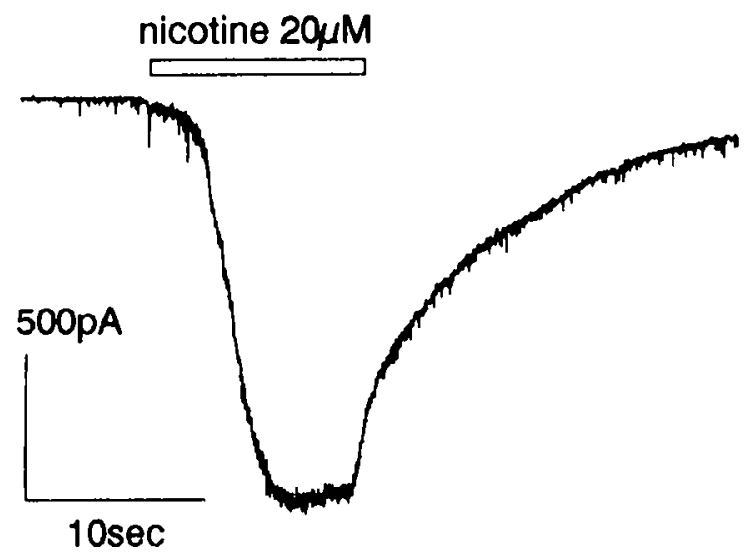

Figure 1. Nicotine enhances the frequency of PSCs in slices of IPN: whole-cell recordings performed in the dorsal part of the IPN. Holding potential, $-80 \mathrm{mV}$. A1, PSC frequency increases after application of nicotine $(1 \mu \mathrm{M})$. $A 2$, PSC frequency during successive applications of increasing concentrations of nicotine. The effect of nicotine is observed at low micromolar concentrations of agonist and is dose dependent. $A l$ and $A 2$ are from the same neuron. Bin, $2 \mathrm{sec}$. $B$, At low concentrations, nicotine has mainly a presynaptic effect $(B I)$, while at higher concentrations, nicotine activates a large inward PSC (B2). B1 and $B 2$ are from the same neuron.

rather than indirectly by postsynaptic stimulation and generation of action potentials. Activation of presynaptic $\mathrm{nAChR}$ could lead to calcium influx in the synaptic bouton either directly though the nicotinic channel itself (Mulle et al., 1992; Vernino et al., 1992) or indirectly by activation of voltage-dependent calcium channels, although neither of these propositions have been demonstrated experimentally.

Our purpose was to examine in some detail the presynaptic action of nicotinic agonists on synaptic function in the IPN. We and others have previously demonstrated the presence of both pre- and postsynaptic nAChRs in this region (Brown et al., 1984; Clarke et al., 1986; Mulle et al., 1991). In this article, we describe the action of nicotinic agonists on the synaptic activity of IPN neurons using whole-cell recordings on thin slices and on a preparation of freshly isolated neurons that have retained synaptic terminals attached to their cell body. Our results suggest that, in the IPN, nAChRs can operate at a preterminal level, that is, on the terminal portion of axons. Activation of these "preterminal" receptors can lead to spike discharge and generalized transmitter release.

\section{Materials and Methods}

Brain slices. Slices of IPN $(120 \mu \mathrm{m})$ were prepared from $17-26 \mathrm{~d}$ postnatal Sprague-Dawley rats as described by Edwards et al. (1989). The various subdivisions of the IPN (Hamill and Lenn, 1983) could be easily distinguished using an upright microscope equipped with Hoffmann differential interference contrast (Leica, Rueil-Malmaison, France).
Solutions and drugs. Experiments were performed at room temperature $\left(22-24^{\circ} \mathrm{C}\right)$. Slices were continuously superfused using an extracellular medium composed of $125 \mathrm{~mm} \mathrm{NaCl}, 2.5 \mathrm{~mm} \mathrm{KCl}, 26 \mathrm{~mm}$ $\mathrm{NaHCO}_{3}, 1.25 \mathrm{~mm} \mathrm{NaH}_{2} \mathrm{PO}_{4}, 2 \mathrm{~mm} \mathrm{CaCl}, 1 \mathrm{~mm} \mathrm{MgCl}, 25 \mathrm{~mm}$ glucose bubbled with $95 \% \mathrm{O}_{2}, 5 \% \mathrm{CO}_{2}(\mathrm{pH} 7.4)$. The osmolarity was adjusted to 320 mOsm with sucrose. Drugs were superfused either through parallel input to the perfusion chamber or using an array of four tubes that could be moved laterally and that permitted changes of the solution surrounding the neuron in less than $1 \mathrm{sec}$.

Freshly isolated neurons. The procedure has been described in a previous study (Mulle et al., 1991). Briefly, the brains of 15-20-d-old rats were removed and placed in a cold PIPES-based saline bubbled with $100 \% \mathrm{O}_{2}$. The IPN was dissected under a binocular microscope. After enzymatic treatment with papain $\left(40 \mathrm{~min}\right.$ at $35^{\circ} \mathrm{C}$ in $10 \mathrm{ml}$ of PIPES saline containing $50 \mathrm{U}$ papain and $0.2 \mathrm{~mm}$ calcium), and gentle trituration with large-bore Pasteur pipettes, cells were plated onto glass coverslips in Dulbecco's modified Eagle's medium containing $25 \mathrm{~mm}$ HEPES and supplemented with IJltroser G (2\%) (IBF, Villneuve-laGarenne, France). Cells were kept in an incubator at $37^{\circ} \mathrm{C}$ and used on the same day. The only difference with the former protocol is the concentration of calcium used $(0.2 \mathrm{~mm})$ during the enzymatic treatment and the very gentle trituration. Synaptic activity on acutely dissociated mammalian neurons that had retained attached synaptic boutons has already been described using slightly different procedures (Drewe et al., 1988).

Whole-cell recordings. The solution in the patch pipette contained, unless stated otherwise, $135 \mathrm{mM} \mathrm{CsCl}, 1 \mathrm{~mm} \mathrm{MgCl}, 0.5 \mathrm{~mm} \mathrm{CaCl}, 10$ mM EGTA, 2 mм Na-ATP, $10 \mathrm{~mm}$ HEPES and was buffered to pH 7.2 with $\mathrm{CsOH}$. In some experiments $\mathrm{CsCl}$ was substituted with $\mathrm{Cs}$-methanesulfonate. Mechanical cleaning of the slice was not necessary for obtaining gigaseals; it was sufficient to apply positive pressure while advancing through the tissue. Whole-cell recordings were performed (pipette resistance, 2-5 M $\Omega$ ) using an Axopatch ID (Axon Instruments, 


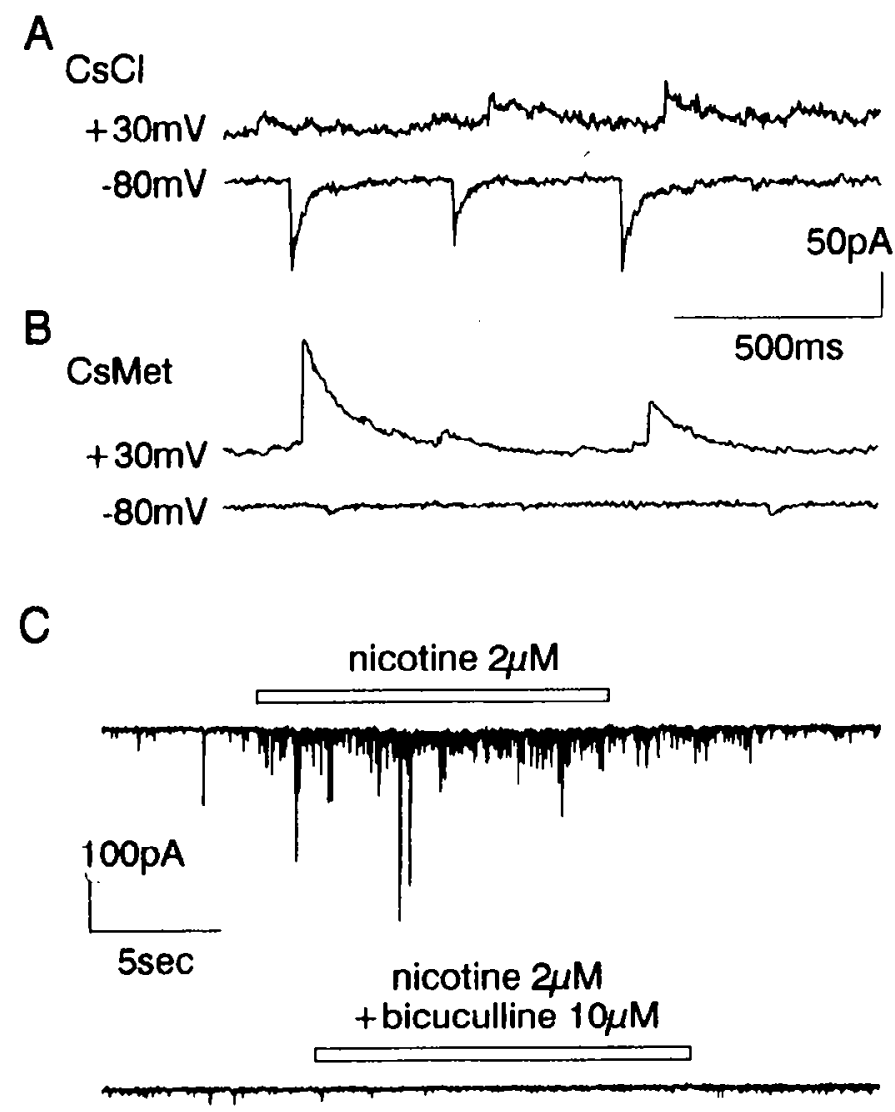

Figure 2. Nicotine enhances the frequency of GABAergic PSCs in slices of IPN. $A$ and $B$, The PSCs activated by nicotine are carried by chloride ions. Recording of synaptic activity during application of nicotine $(1 \mu \mathrm{M})$ at positive and negative potentials. The patch pipette contained $\mathrm{Cl}^{-}$as the main anion in $A$. In $B$, it contained methanesulfonate an impermeant anion. $C$, The action of nicotine is abolished in the presence of bicuculline, a GABA , antagonist. Holding potential, -80 mV.

Burlingame, CA). Unless otherwise mentioned, the membrane potential was held at $-80 \mathrm{mV}$.

Data analysis. Currents were monitored with an Axopatch ID (Axon Instruments), filtered at $2 \mathrm{kHz}$, stored on a Biologic DAT, fed into a computer at an acquisition rate of $100 \mu \mathrm{sec}$ per point with the program pCLAMP, and further analyzed with software written in TurboPascal. The postsynaptic currents (PSCs) were detected using a fast rise time criterion and were submitted to a visual control. The amplitude distributions were compared using cumulative diagrams and the Kolmogorov-Smirnov test (sTATGRAPH, Graphic Software Systems, Inc.). Significant differences were considered to be those with the approximate significant value less than 1\% (see Cohen et al., 1992; Manabe et al., 1992).

Immunohistochemistry of isolated neurons. Freshly isolated neurons were fixed with a methanol-acetic acid $(95: 5)$ mixture for $10 \mathrm{~min}$ at $-20^{\circ} \mathrm{C}$. They were then washed twice with PBS and incubated with a monoclonal antibody against synaptophysin (1:100; Amersham), in the presence of gelatin $(2.5 \%)$ and Triton $(0.1 \%)$. The preparation was then washed in PBS, reacted for $1 \mathrm{hr}$ with a biotinylated donkey anti-mouse IgG (1:200; Vector), washed again in PBS, and kept for $\mathrm{l} \mathrm{hr}$ in streptavidin fluorescein isothiocyanate (FITC; $1: 200$; Amersham). After extensive washing, the preparation was mounted with Mowiol (Hoechst).

Confocal visualization. Preparations were examined with a confocal microscope (Phoibios 1000, Molecular Dynamics). Excitation was obtained with an argon laser set at $488 \mathrm{~nm}$ for FITC excitation, and the emitted light was filtered with an appropriate long-pass filter $(515 \mathrm{~nm})$. The background noise was reduced and the contrast was enhanced by applying a median $(3 \times 3 \times 3)$ Gaussian filter to the original data. The gray coding for fluorescence emission was obtained with a linear lookup table.

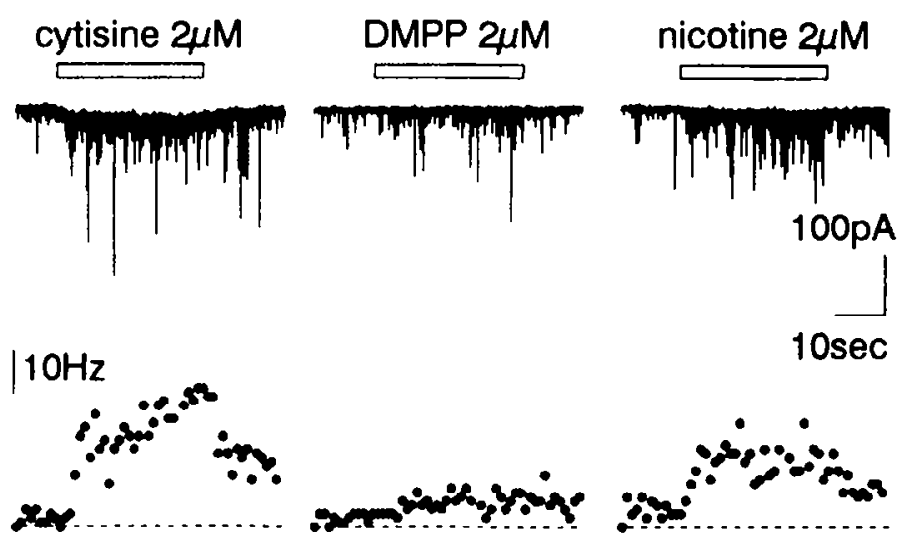

Figure 3. Comparison of the effects of nicotinic agonists on the frequency of PSCs in slices of IPN: traces of current (top) and corresponding diagrams of PSC frequency (botlom) (bin, $1 \mathrm{sec}$ ). Holding potential, -80 $\mathrm{mV}$.

\section{Results}

Activation of $n A C h R$ s increases the frequency of $G A B A$ ergic $P S C$ s in thin slices of IPN

Over 150 whole-cell recordings were performed in the rostral nucleus and in the dorsal part of the central and intermediate nuclei of the IPN that were shown to display the most dense ${ }^{3} \mathrm{H}$-nicotine labeling (Clarke et al., 1986). Spontaneous PSCs occurred in most neurons with variable frequency. In most neurons, PSCs were abolished in the presence of bicuculline and were not observed when methanesulfonate ions, which do not permeatc anion channels, were substituted for $\mathrm{Cl}^{-}$in the pipette solution. This indicates that the PSCs were due to the activation of GABAergic afferents. The amplitude of the PSCs was variable and ranged from $10 \mathrm{pA}$ to several hundreds of picoamperes with a mean value of $29.3 \pm 8.5 \mathrm{pA}$ ( $n-15$ neurons). These PSCs were also characterized by their slow decay. When measured on four neurons, the half-decay time was $11.4 \pm 3.5 \mathrm{msec}$ (range, 4-32.9 msec). The GABAergic PSCs could easily be distinguished from small and fast spontaneous PSCs that were observed in only a small number of neurons ( 8 out of 171 neurons). These PSCs were blocked by 6-cyano-7-dinitroquinoxaline-2,3dione (CNQX; $10 \mu \mathrm{M}$ ) and thus probably represented glutamatergic PSCs. The frequency of both types of PSCs was greatly increased by addition of 4-aminopyridine (4-AP; $10 \mu \mathrm{M}$ ) probably through depolarization of nerve terminals (data not shown). PSCs evoked by 4-AP were totally blocked by a solution containing CNQX and bicuculline, such that no PSCs other than glutamatergic or GABAergic ones were present on these neurons. In particular, we found no evidence for a block of spontaneous PSCs by nicotinic antagonists under these conditions.

Application of nicotine evoked within less than $1 \mathrm{sec}$ an increase in frequency of PSCs in a large majority of IPN neurons in the rostral, central and intermediate nuclei (119 out of 134 neurons). This effect was dose dependent and could be observed at submicromolar concentrations of nicotine (Fig. $1 A, B$ ). In agreement with our previous work on acutely isolated IPN neurons (Mulle et al., 1991), higher concentrations of nicotine elicited an inward current duc to activation of postsynaptic nAChRs (Fig. 1B2). All neurons $(n=54)$ tested with a nicotine concentration above $10 \mu \mathrm{M}$, which showed a postsynaptic response, also exhibited an increase in the frequency of PSCs. In most 
A

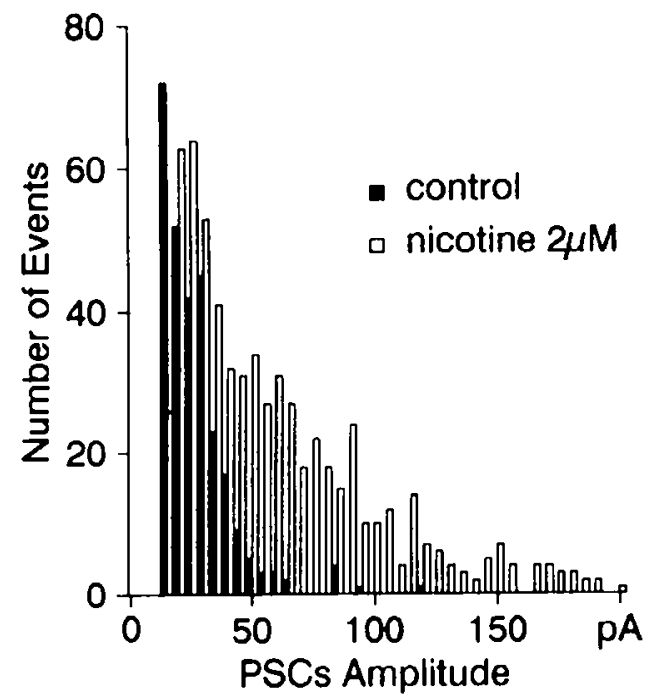

B
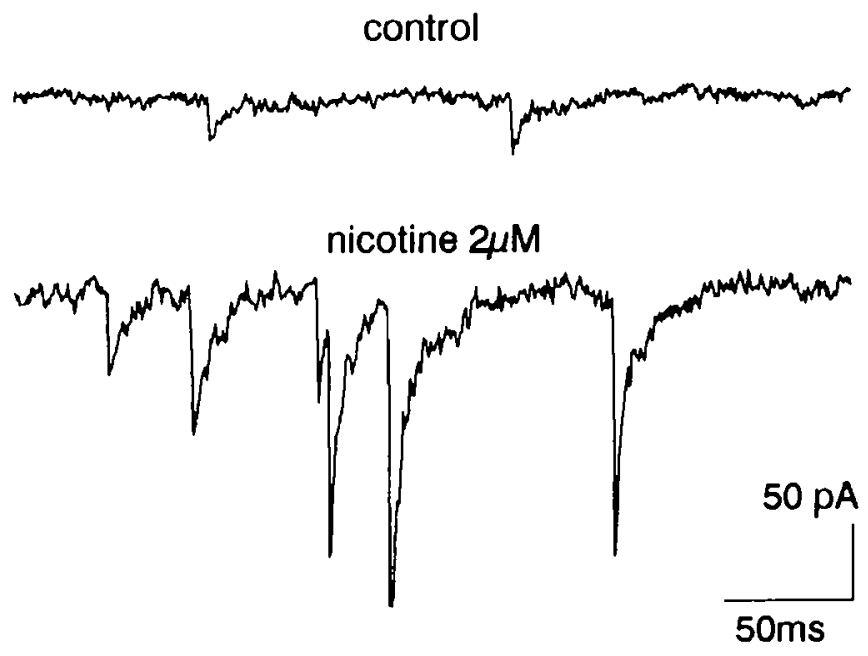

C
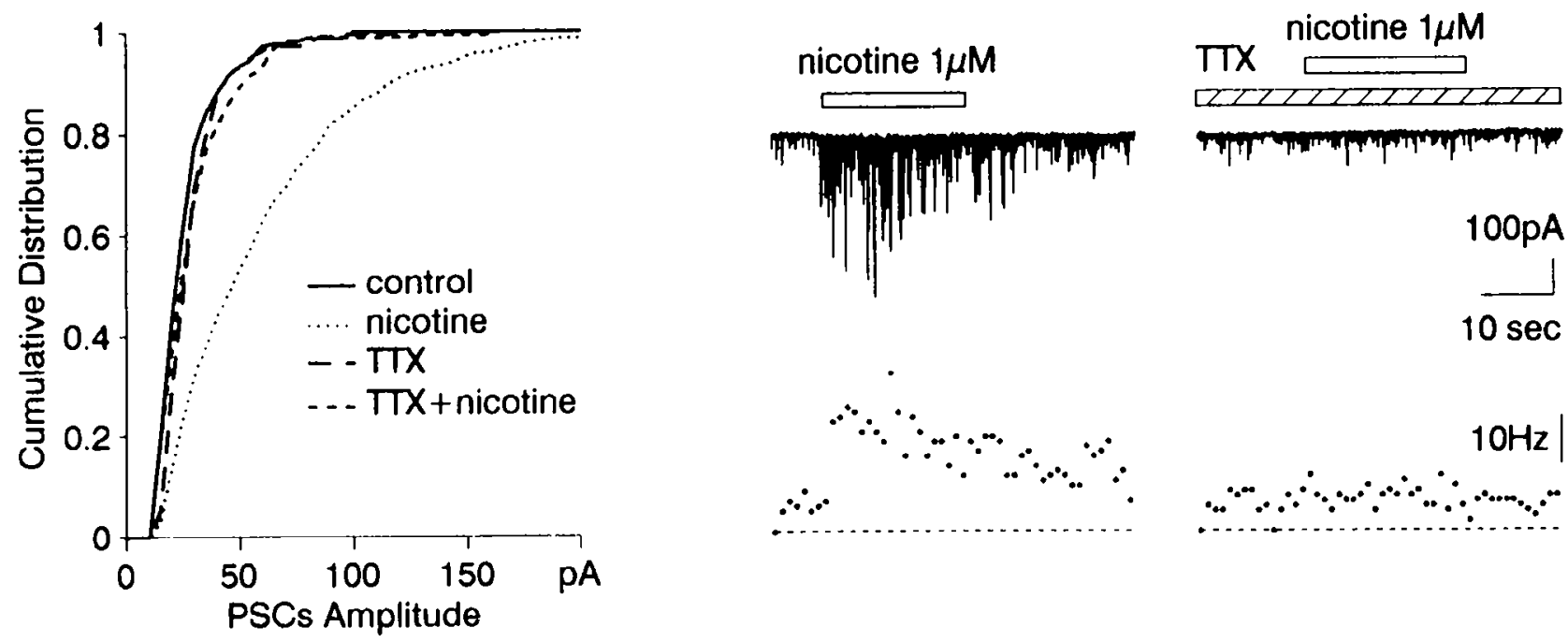

$10 \mathrm{sec}$

Figure 4. Effects of nicotine and TTX on the amplitude and the frequency of PSCs in slices of IPN. A, Histogram of the amplitude of PSCs recorded for $15 \mathrm{sec}$ before (solid bars) and during (open bars) application of nicotine $(2 \mu \mathrm{M})$. The smallest events could not be detected under nicotine because of the postsynaptic response. Bin, $5 \mathrm{pA}$. $B$, Current trace recorded before and during application of nicotine. $C$, Cumulative distribution of PSC amplitude under control conditions, during application of $2 \mu \mathrm{M}$ nicotine, $1 \mu \mathrm{M} \mathrm{TTX}$, or $2 \mu \mathrm{M}$ nicotine $+1 \mu \mathrm{M}$ TTX. Using the Kolmogorov-Smirnov test, no significant differences can be observed between control, TTX, and TTX + nicotine conditions (see Materials and Methods). $D$, TTX $(2 \mu \mathrm{M})$ suppresses the presynaptic effect of nicotine in slices of IPN: traces of current (top) and corresponding diagrams of PSC frequencies (bottom) (bin, $1 \mathrm{sec}$ ). Holding potential, $-80 \mathrm{mV}$.

neurons, the presynaptic effect could be observed at concentrations of agonist that did not activate postsynaptic nAChRs.

In a large majority of neurons, PSCs triggered by nicotine showed a slow decay phase (see Fig. 4). They reversed around $0 \mathrm{mV}$ when $\mathrm{CsCl}$ was present in the patch pipette, but they could only be observed at positive membrane potentials when $\mathrm{Cl}^{-}$ ions were replaced with methanesulfonate (Fig. $2 A, B$ ). Finally, bicuculline $(10 \mu \mathrm{M})$ suppressed all the spontaneous activity evoked by an application of nicotine $(10 \mu \mathrm{M})$ (Fig. $2 C)$. These results demonstrate that nicotine triggers the discharge of GABAergic PSCs. It must be noted, however, that in a few neurons ( $n=5$ out of 134), nicotine $(<2 \mu \mathrm{M})$ triggered PSCs that displayed a rapid decay phase and that could be blocked by CNQX
$(10 \mu \mathrm{M})$, suggesting that these PSCs were glutamatergic. These neurons were not further analyzed.

The effects of nicotine were mimicked by cytisine and dimethylphenyl pyserazinium (DMPP) at comparable concentrations (Fig. 3). The relative order of potency of these agonists was evaluated by measuring their effect on the frequency of PSCs (Fig. 3). At a concentration of $2 \mu \mathrm{M}$ the ratio of frequency increase was cytisine:nicotine $=1.1 \pm 0.5(n=5)$ and DMPP: nicotine $=0.3 \pm 0.1(n=4)$. Stimulation of PSCs by ACh (in the presence of atropine, a blocker of muscarinic receptors) could only be achieved with higher concentrations of agonist. However, after preincubation with eserine $(10 \mu \mathrm{M})$, an anticholinesterase agent, ACh reproduced the effects of nicotine at micro- 
A
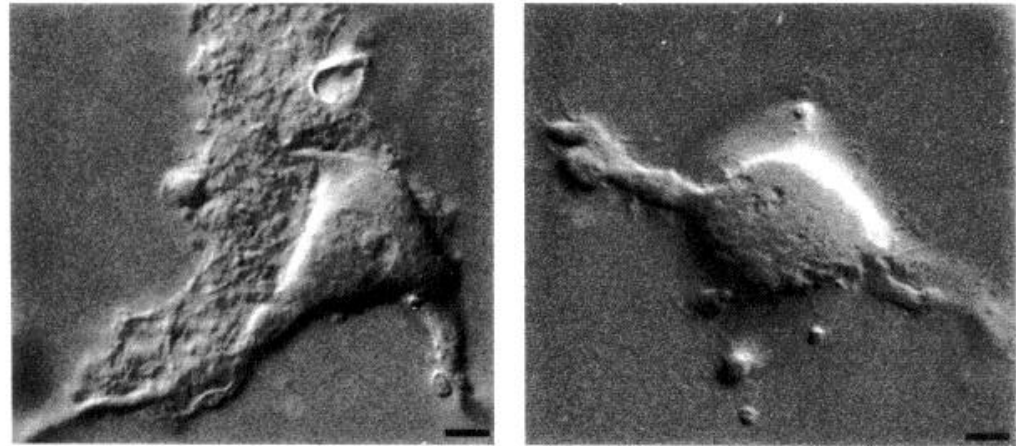

B
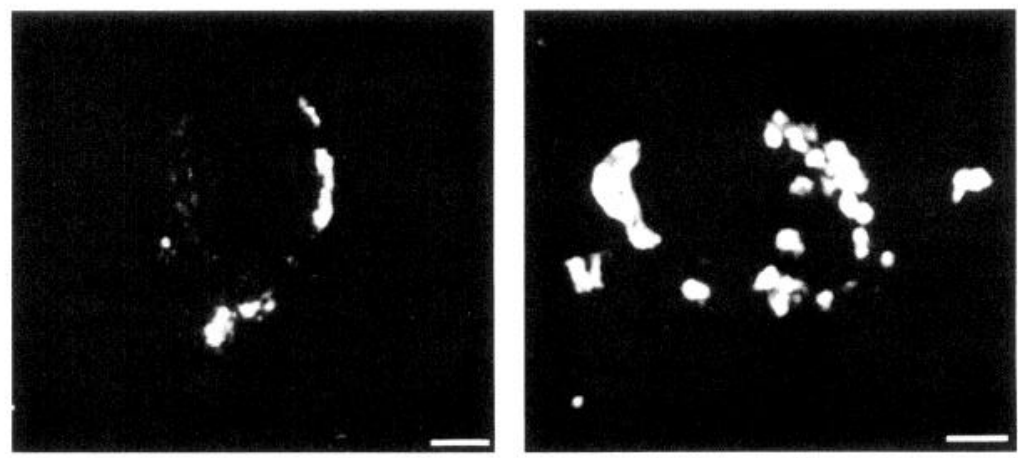

C

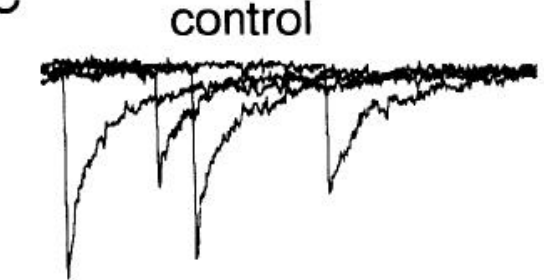

+ bicuculline $10 \mu \mathrm{M}$

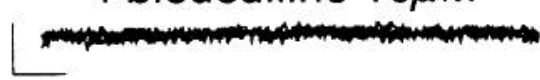

$\mathrm{D}$

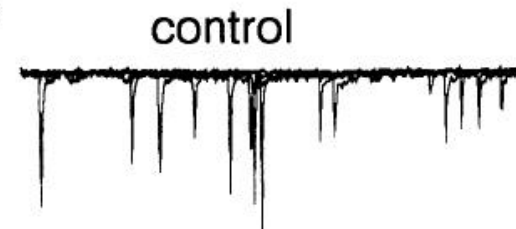

+ CNQX $10 \mu \mathrm{M}$

Figure 5. Freshly dissociated neurons from the IPN retain GABAergic and glutamatergic synaptic terminals. $A$, Nomarski photomicrographs of freshly isolated IPN neurons $1 \mathrm{hr}$ after plating. On the left photomicrograph, note the presence of neuropil surrounding the neuronal cell body. $B$, Confocal images of isolated neurons IPN neurons stained with a monoclonal antibody against synaptophysin. The left image is an optical confocal section of labeled neuron, and the right image is a look through projection of 15 sections, allowing the visualization of the cell surface. Note the presence of clusters of dense labeling surrounding the cell bodies. $C$ and $D$, Whole-cell recordings from two different freshly isolated IPN neurons. Holding potential, $-80 \mathrm{mV}$. $C$, Samples of spontaneous PSCs blocked by bicuculline, a GABA $\mathrm{A}_{\mathrm{A}}$ antagonist. $D$, Samples of spontaneous PSCs blocked by CNQX, an antagonist of non-NMDA glutamate receptors. Scale bars: $4 \mu \mathrm{M}$. Calibration: $C, 10 \mathrm{msec}, 30 \mathrm{pA} ; D, 10 \mathrm{msec}, 10 \mathrm{pA}$.

molar concentrations (ratio ACh:nicotine $=0.8 \pm 0.3, n=3$ ). The effect of nicotine ( $2 \mu \mathrm{M})$ on PSC frequency was completely blocked by mecamylamine $(10 \mu \mathrm{M})$, hexamethonium $(100 \mu \mathrm{M})$, and dihydro- $\beta$-erythroidine $(10 \mu \mathrm{M})$, but was not affected by neuronal bungarotoxin (at a concentration of $200 \mathrm{~nm}$ for 45 $\mathrm{min}$ ) or by $\alpha$-bungarotoxin (at a concentration of $500 \mathrm{nM}$ for 15 $\min )$.

\section{Mechanism of action of nAChRs}

We have attempted to clarify the mechanism by which nAChRs increase the discharge of GABAergic PSCs. As shown in Figure 1 , the main effect of $2 \mu \mathrm{M}$ nicotine on GABAergic PSCs is a strong increase of their frequency. The distribution of PSC amplitude was shifted under nicotine toward larger values in 9 out of 13 cells (Fig. $4 A-C$ ) but remained unchanged in the four other cells. Nicotine could thus elicit a class of large PSCs that were rarely seen under control conditions.

Perfusing the slice with $1 \mu \mathrm{M}$ TTX slightly decreased the frequency of spontaneous PSCs (decrease of $30 \% ; n=6$ neurons) while the distribution of the amplitude of the PSCs was not changed in five out of seven neurons (and was slightly shifted toward smaller values in two out of seven neurons). This suggested that synaptic activity recorded under control conditions was mainly due to spontaneous exocytosis. Moreover, TTX completely suppressed (five out of seven neurons) or largely depressed (two out of seven neurons) the effect of $2 \mu \mathrm{M}$ nicotine on the frequency and on the distribution of the amplitude of the PSCs (Fig. 4C,D). The large PSCs elicited by nicotine were thus most likely triggered by action potentials, indicating that the nAChRs are acting upstream to the synaptic boutons rather than on the synaptic boutons.

This finding could suggest that nicotine might act to increase firing of GABAergic interneurons present in the slice. However, as stated above, nicotinic agonists could trigger synaptic discharge at a concentration where no nicotinic PSCs were detected. Moreover, the experiments described below on isolated IPN neurons strongly support an alternative explanation.

\section{Presynaptic nicotinic action on acutely isolated IPN neurons}

In a previous study we have described the properties of postsynaptic nAChR on IPN neurons utilizing a preparation of freshly isolated neurons (Fig. 5A). Under conditions of low external calcium $(0.2 \mathrm{~mm})$ and gentle pipette trituration, the same dissociation procedure yielded neurons that retained synaptic terminals, as already described using another dissociation procedure (Drewe et al., 1988). We provide both immunocytochemical and physiological evidence for the presence of remaining synaptic terminals on isolated IPN neurons.

First, we stained the dissociated neurons with an antibody against synaptophysin, an integral protein of synaptic vesicles. We observed clusters of dense labeling surrounding numerous cell bodies of the IPN neurons, as illustrated on a confocal image (Fig. $5 B$ ). Then, over 50 of these neurons showed synaptic like activity when recorded in the whole-cell mode (Fig. $5 C, D$ ). In 
A
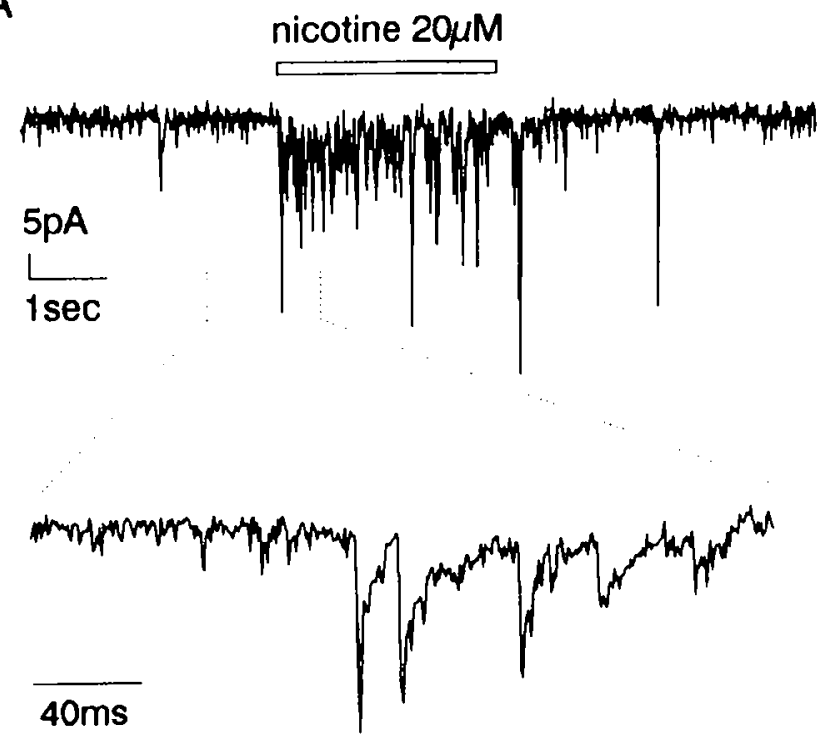

B

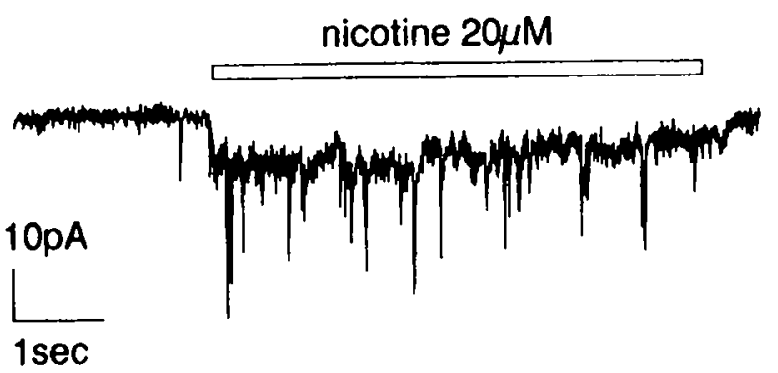

Figure 6. Effects of nicotine on synaptic activity recorded in freshly isolated IPN neurons. $A$ and $B$, Two examples of current recordings from neurons responding to nicotine by an increased PSC frequency. In $B$, nicotine also activates an inward current due to activation of postsynaptic nAChRs. Holding potential, $-80 \mathrm{mV}$.

most neurons the PSCs had a slow phase of decay (Fig. $5 C$ ), reversed around $0 \mathrm{mV}$ under conditions of symmetric $\mathrm{Cl}$ concentration, and could be blocked by bicuculline $(10 \mu \mathrm{M})$, demonstrating that they were due to the activation of GABAergic terminals. In only a few neurons $(n=3)$ could we observe very fast rising and fast decaying PSCs, that were blocked by CNQX $(10 \mu \mathrm{M})$ (Fig. $5 D)$, and were thus due to the activation of glutamatergic synaptic terminals.

The frequency of the PSCs was increased by external application of a medium containing $10 \mathrm{mM} \mathrm{KCl}$ (not shown). This increase, as well as spontaneous activity, was largely depressed by superfusion with TTX $(1 \mu \mathrm{M})$, suggesting that the spontancous synaptic activity was due to the generation of action potentials in terminal axon branches that had remained attached to the neuronal cell body. This correlated well with the fact that amplitude histograms constructed for GABAergic events showed a wide distribution incompatible with that of miniature GABAcrgic PSCs (Edwards et al., 1990).

Addition of nicotine evoked in 29 neurons a rapid increase in the frequency of PSCs (Fig. 6). This increase was often accompanied with an activation of a postsynaptic nicotinic response (Fig. 6B). The concentrations of nicotine used on the dissociated neurons were higher than those used on the thin slices, in order to increase the probability of presynaptic re- sponses to nicotine. When the postsynaptic nicotinic response was large, noise increased and presynaptic activity was difficult to detect, but the PSCs could readily be observed as outward currents at positive membrane potentials (see Fig. 7A), while the postsynaptic response was abolished, due to the rectification properties of the nAChRs (Mulle et al., 1991).

This effect could be mimicked by other nicotinic agonists such as ACh (Fig. $7 A$ ) or cytisine. It was blocked by mecamylamine (10 $\mu \mathrm{M})$ (Fig. $7 B)$ and $d$-tubocurarine $(10 \mu \mathrm{M})$. These results indicate that the increase in PSC frequency was due to the activation of an $\mathrm{nAChR}$, and they correlate well with results obtained on thin slices.

As suggested by the time course of decay of the PSCs, as well as the reversal around $0 \mathrm{mV}$ in symmetric $\mathrm{Cl}$ conditions, the PSCs activated by nicotine were blocked by bicuculline (Fig. $8 A$ ) and were thus likely to be GABAergic PSCs. In the absence of calcium in the external medium, spontaneous and nicotineenhanced PSCs were not observed. Finally, the effect of nicotine on PSC frequency was completely abolished (four out of six neurons) or largely depressed by superfusion with TTX (Fig. $8 B$ ): in two neurons, we found a residual effect of nicotine after blockade of $\mathrm{Na}^{+}$spikes with TTX.

\section{Discussion}

In the long-term perspective of understanding the role of $\mathrm{nAChRs}$ in the function of synaptic circuits in the CNS, we have studied the effects of nicotinic agonists on the synaptic activity of neurons in the IPN. We show that activation of $\mathrm{nAChRs}$ by nicotinic agonists in the low micromolar range triggers the discharge of GABAergic PSCs. We present evidence that at least part of the $\mathrm{nAChRs}$ involved in this action are located on the terminal part of GABAergic axon terminals. These $n A C h R s$ can be designated as preterminal nAChRs (Wessler, 1992). In contrast, nAChRs located close to terminal boutons are referred to as presynaptic nAChRs.

The IPN is of particular interest for the study of the role of $\mathrm{nAChRs}$ in the function of synaptic circuits in the CNS for the following reasons. The IPN contains an abundance of cholinergic markers, possessing for instance a high concentration of ChAT immunoreactivity (Houser et al., 1983). Cholinergic innervation is wholly extrinsic and derives principally from the medial habenula and the mediobasal forebrain through the fasciculus retroflexus (see Woolf and Butcher, 1985, for review). Putative nAChRs in the IPN have been labeled with $\alpha$-bungarotoxin, ${ }^{3} \mathrm{H}-\mathrm{ACh}$ (with excess muscarine), ${ }^{3} \mathrm{H}$-nicotine (Clarke et al., 1985), and antibodies raised against nAChRs (Deutch et al., 1987; Swanson et al., 1987). Consistent with the existence of cholinoceptive binding sites, in situ hybridization studies have revealed a high degree of expression of several genes encoding subunits of the neuronal nAChR family; the IPN has the highest expression of the $\alpha 2$ subunit gene in the CNS; it also shows moderate to high expression of $\alpha 3, \alpha 4, \alpha 5, \beta 2$, and $\beta 4$ subunits (Wada et al., 1989; Boulter et al., 1990). Finally, electrophysiological studies performed on slices (Brown et al., 1983) or on acutely isolated neurons (Mulle et al., 1991) have demonstrated the existence of functional $\mathrm{nAChRs}$ on IPN neurons. Using patch-clamp techniques and acutely isolated IPN neurons, we had shown that $\mathrm{nAChR}$ located at the level of the neuronal cell bodies differed from other nAChRs in several of their pharmacological and electrophysiological properties. Comparison of the order of potency of several agonists to that obtained for nAChR expressed in Xenopus oocytes (Luetje and Patrick, 1991), 
A1

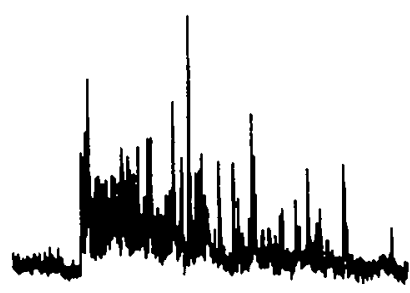

nicotine $20 \mu \mathrm{M}$

B1

Figure 7. Pharmacological characteristics of the presynaptic action of nicotine on freshly isolated IPN neurons. $A$, The effect of nicotine $(20 \mu \mathrm{M})$ is $\mathrm{mim}$ icked by ACh $(20 \mu \mathrm{M})$. Recording was made at a positive potential $(+40 \mathrm{mV})$ because at negative potentials the presynaptic activity was masked by a large nicotinic inward current. $B$, The presynaptic action of nicotine is blocked by mecamylamine. Holding potential, $-80 \mathrm{mV} . A$ and $B$ are from two different neurons.
$\mathrm{A} 2$

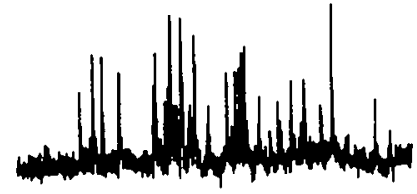

ACh $20 \mu \mathrm{M}$

B2

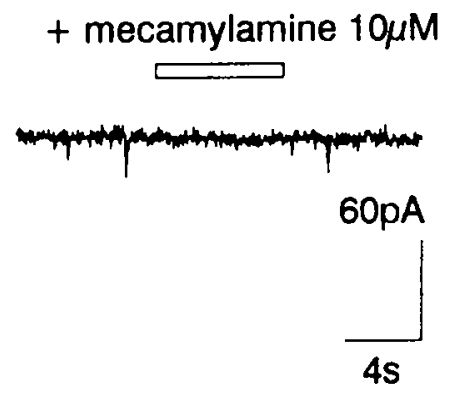

suggested that this IPN nAChR may consist of $\alpha 2$ and $\beta 4$ subunits. Given the diversity of cell types and of $n A C h R s$ in the IPN, it is, however, quite possible that several types of $n A C h R$ coexist in the IPN. Electrophysiological studies (Brown et al., 1983; Mulle et al., 1991) have shown that field potentials evoked by stimulation of the fasciculus retroflexus were affected by superfusion with nicotinic agonists. In agreement with anatomical studies combined with lesion of the fasciculus retroflexus (Clarke et al., 1986), these studies strongly suggested that some of the nAChRs in the IPN are located on these afferent terminals.

Most of the evidence that presynaptic nAChRs play an important part in the CNS comes from study of transmitter release using synaptosomal preparations and brain slices. The use of TTX provides a strong argument for the location of $\mathrm{nAChR}$ at presynaptic sites, that is, on synaptic boutons. There is some agreement, although it has not been demonstrated, that the activation of these $\mathrm{nAChRs}$ leads to depolarization of the nerve terminal, subsequent calcium entry, and transmitter release. Only few studies have addressed the question of the function of presynaptic nAChRs using electrophysiological methods. We have attempted to detail the presynaptic action of nicotine in the IPN using patch-clamp techniques and thin slices.

In a large majority of neurons recorded in the dorsal region of the IPN, and that corresponded roughly (nomenclature of Hamill and Lenn, 1984) to the rostral, central and intermediate parts of the IPN, nicotinic agonists elicited a rapid increase in the frequency of GABAergic PSCs. This action was mediated by the activation of a receptor that displayed the pharmacological properties of a neuronal $\mathrm{nAChR}$. This $\mathrm{nAChR}$ was activated by nicotinic agonists (such as nicotine, cytisine, and DMPP) acting on $\mathrm{nAChR}$ in ganglionic and CNS neurons as well as on neuronal $n A C h R s$ expressed in oocytes (Mulle and Changeux, 1990; Luetje and Patrick, 1991 ; Mulle et al., 1991). This nAChR was blocked by classical nicotinic antagonists (hexamethonium, dihydro- $\beta$-erythroidine, and mecamylamine). These features were found on the thin-slice preparation as well as on acutely isolated neurons.

The key issue of this study was to understand the mechanism of the presynaptic action of nicotine on the GABAergic afferents.

A
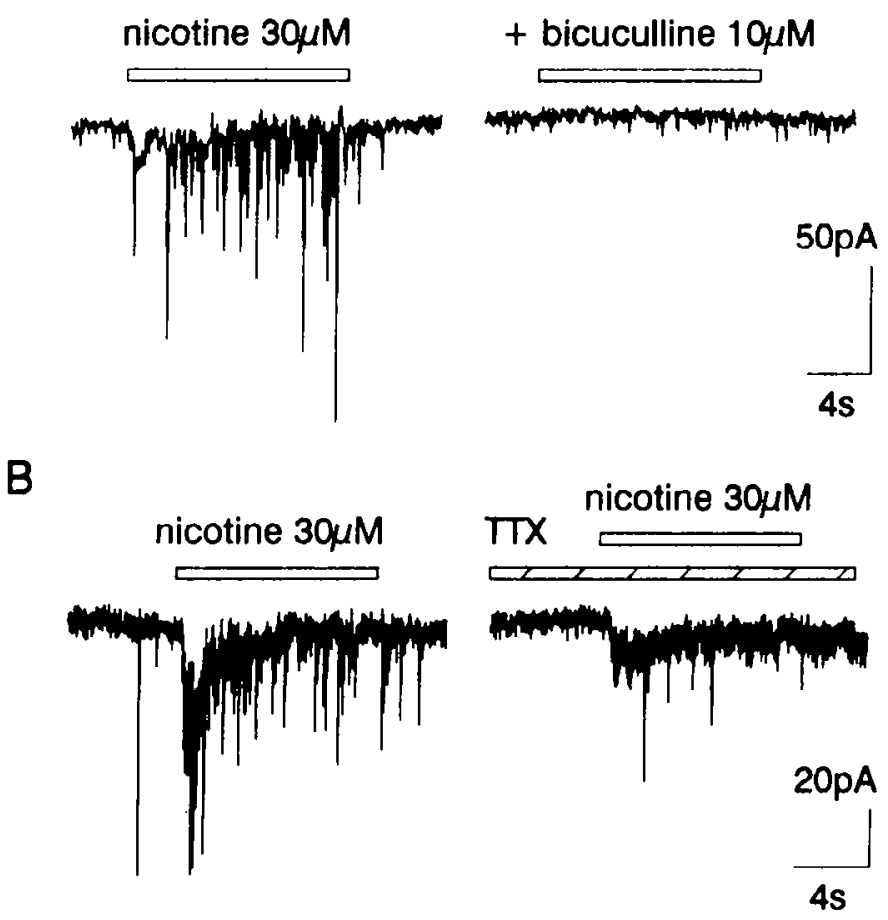

Figure 8. Mechanism of the presynaptic action of nicotine on freshly isolated IPN neurons. $A$, Nicotine activates GABAergic synaptic currents: its effect is blocked by bicuculline. $B$, TTX $(1 \mu \mathrm{M})$ largely depresses the presynaptic effect of nicotine. $A$ and $B$ are from different cells. Holding potential, $-80 \mathrm{mV}$. 
Studies on synaptosomes from several brain regions have shown that nicotine could activate the release of neurotransmitter in a TTX-independent manner (see Wonnacott et al., 1990, for review), thus suggesting a direct link between $n A C h R$ activation and cytosolic calcium elevation associated with the transmitter release process. In contrast, the presynaptic action of nicotine that we have described in the IPN was blocked by TTX. This effect could be attributed to an activation of nAChRs located on the somatodendritic region of intrinsic neurons, and subsequent triggering of action potentials. However, we show that the presynaptic action of nicotine observed in IPN thin slices can be reproduced using a preparation of isolated neurons that had retained synaptic contacts. In this preparation, neurons are physically isolated from one another, in such a way that the synaptic activity that we recorded could only be attributed to the activation of terminal axonal afferents attached to the cell body. In this preparation, the increase in PSC frequency by nicotinic agonists was also blocked by TTX, indicating that the nAChRs involved are different in their location from the presynaptic $n A C h R s$ described in synaptosomal preparations. These experiments thus suggest the presence of "preterminal" nAChRs, located on terminal regions of the axon but not in the proximity of a single synaptic bouton. Our data suggest that activation of these nAChRs directly triggers TTX-sensitive spikes in the terminal portion of the GABAergic axons. These action potentials presumably initiate synchronous GABA release from multiple presynaptic terminals.

We do not exclude that part of the effect of nicotinic agonists observed in thin slices could be due to the activation of postsynaptic nAChRs located on the soma or dendrites of intrinsic IPN neurons. However, nicotinic agonists show a presynaptic action at concentrations roughly 10 times lower than those necessary to activate a postsynaptic response in these IPN neurons. This finding suggests that preterminal and somatic nAChRs might be different subtypes of $\mathrm{nAChRs}$, and that at low micromolar concentrations of agonist, only the preterminal nAChRs are activated. The order of potency of nicotinic agonists for the preterminal action is in the order cytisine $=$ nicotine $=\mathrm{ACh}$ (in the presence of eserine) > DMPP. However, cytisine is about threefold more potent than DMPP. In contrast, for postsynaptic nAChRs, cytisine is about 30 times more potent than DMPP (Mulle et al., 1991). Thus, these findings would be in favor of the hypothesis that the postsynaptic and the preterminal actions are not mediated by the same subtype of nAChR. However, this remains to be firmly established by, for instance, the use of subunit-specific antibodies.

How do these preterminal nAChRs affect the function of synaptic circuits in the IPN? A large population of glutamic acid decarboxylase (GAD)-containing neuronal cell bodies have been described in the IPN (Kawaja et al., 1989), and most, if not all, GABAergic afferents arise from these intrinsic neurons. It is thus most likely that the preterminal nAChRs are not located on fasciculus retroflexus fibers, although these fibers have been shown to possess presynaptic nAChRs in various anatomical and physiological studies (Brown, 1984; Clarke et al., 1986; Mulle et al., 1991). GABAergic neurons in the IPN are likely to express the preterminal $\mathrm{nAChRs}$ on their local axon terminals. We do not know whether these GABAergic neurons also possess the postsynaptic nAChRs found on acutely isolated neurons and in the thin-slice preparation. However, this seems unlikely since the regions from which most recordings were made do not coincide with the regions of high density of GAD- positive cell bodies. Cholinergic afferents arise principally from the medial habenula through the fasciculus retroflexus (Constetabile et al., 1987; Eckenrode et al., 1987), and terminate in the rostral, central, and intermediate subnuclei. However, no axoaxonic synaptic contacts have been observed between fascicular axons and GAD-positive terminals (Kawaja et al., 1989). This raises the question of how $\mathrm{ACh}$ released from a cholinergic axon terminal will reach the $\mathrm{nAChRs}$ located on the terminal portion of the axon. These preterminal receptors could respond to ACh that accumulates extrasynaptically after periods of intense synaptic activity or to ACh released in a paracrine manner (see Fuxe and Agnati, 1991). It is worth mentioning that the high sensitivity of these receptors to ACh could be compatible with one of these mechanisms despite the activity of AChEs. ACh released either synaptically or in paracrine manner will primarily cause a depolarization of the postsynaptic neuron. This depolarization will be stopped by inhibition due to the nicotinic activation of the local GABAergic axons. The role of preterminal nAChRs will thus be to prevent prolonged depolarizations, although postsynaptic $\mathrm{nAChRs}$ could still be opened. We have already suggested that the concomitant activation of postsynaptic chloride channels that maintain the neuronal membrane hyperpolarized and activation of $\mathrm{nAChRs}$ could result in a large influx of calcium through the nicotinic channel (Mulle et al., 1992).

In conclusion, we propose the existence of several categories of nAChRs in the CNS according to their locations in different parts of the neuron. Postsynaptic nAChRs located in somatodendritic regions cause a general depolarization of the neuron. Presynaptic receptors located on synaptic boutons locally control transmitter release in a TTX-independent manner. Preterminal nAChRs located on terminal axonal branches control transmitter release by a general depolarization of the afferents, either by facilitating spike transmission at axonal bifurcations or by directly triggering an action potential in these axons.

\section{References}

Alkondon M, Albuquerque EX (1991) Initial characterization of the nicotinic acetylcholine receptors in rat hippocampal neurons. J Receptor Res 11:1001-1021.

Boulter J, O'Shea-Greenfield A, Duvoisin R, Connoly J, Wada E, Jensen A, Gardner P, Ballivet M, Deneris E, McKinnon D, Heinemann S, Patrick J (1990) $\alpha 3, \alpha 5$, and $\beta 4$ : three members of the rat neuronal nicotinic acetylcholine receptor-related gene family form a gene cluster. J Biol Chem 265:4472-4482.

Brown DA, Docherty RJ, Haliwell JV (1983) Chemical transmission in the rat interpeduncular nucleus in vitro. J Physiol (Lond) 341:655670.

Brown DA, Docherty RJ, Haliwell JV (1984) The action of cholinomimetic substances on impulse conduction in the habenulo-interpeduncular pathway of the rat in vitro. J Physiol (Lond) 353:101-109.

Clarke PBS, Swartz RD, Paul SM, Pert CB, Pert A (1985) Nicotinic binding in the rat brain: autoradiographic comparison of ${ }^{3} \mathrm{H}$-acetylcholine, ${ }^{3} \mathrm{H}$-nicotine and ${ }^{125} \mathrm{I}$-alpha-bungarotoxin. J Neurosci 5:13071315.

Clarke PBS, Hamill GS, Nadi NS, Jacobowitz DM, Pert A (1986) ${ }^{3} \mathrm{H}-$ nicotine and 125 I-alpha bungarotoxin labeled nicotinic receptors in the rat interpeduncular nucleus of rats. II. Effects of habenular deafferentation. J Comp Neurol 251:407-413.

Cohen GA, Doze VA, Madison DV (1992) Opioid inhibition of GABA release from presynaptic terminals of rat hippocampal interneurons. Neuron 9:325-335.

Constetabile A, Villani L, Fasolo A, Franzoni MF, Gribaudo L, Okredalen $O$, Fonnum $F$ (1987) Topography of cholinergic and substance $P$ pathways in the habenulo-interpeduncular system of the rat. An immunocytochemical and microchemical approach. Neuroscience 21: 251-270. 
Deutch AY, Holliday J, Roth RH, Chun LLY, Hawrot E (1987) Immunohistochemical localization of a neuronal nicotinic acetylcholine receptor in mammalian brain. Proc Natl Acad Sci USA 84:86978701.

Drewe JA, Childs GV, Kunze DL (1988) Synaptic transmission between dissociated adult mammalian neurons and attached synaptic boutons. Science 241:1810-1813.

Eckenrode TC, Barr GA, Battisti WP, Murray M (1987) Acetylcholine in the interpeduncular nucleus of the rat: normal distribution and effects of deafferentation. Brain Res 418:273-286.

Edwards FA, Konnerth A, Sakmann B, Takahashi T (1989) A thin slice preparation for patch-clamp recordings from neurons of the mammalian central nervous system. Pfluegers Arch 414:600-612.

Edwards FA, Konnerth A, Sakmann B, Busch C (1990) Quantal analysis of inhibitory synaptic transmission in the dentate gyrus of rat hippocampal slices: a patch-clamp study. J Physiol (Lond) 430:213249.

Fuxe K, Agnati LF (1991) Two principal modes of electrochemical communication in the brain: volume vs wiring transmission. In: Volume transmission in the brain (Fuxe K, Agnati LF, eds), pp 1-10. New York: Raven.

Giorguieff MF, Le Floch ML, Westfall TC, Glowinski J, Besson MJ (1976) Nicotinic effect of acetylcholine on the release of newly synthesized dopamine in rat striatal slices of cat caudate nucleus. Brain Res 106:117-131.

Hamill GS, Lenn NJ (1983) The subnuclear organization of the rat interpeduncular nucleus: a light and electron microscopic study. J Comp Neurol 222:396-408.

Houser CR, Crowford GD, Barber RP, Salvaterra PM, Vaughn JE (1983) Organization and morphological characteristics of cholinergic neurons: an immunocytochemical study with a monoclonal antibody to choline acetyltransferase. Brain Res 266:97-119.

Kawaja MD, Flumerfelt BA, Hrycyshyn AW (1989) Glutamate decarboxylase immunoreactivity in the rat interpeduncular nucleus: a light and electron microscope investigation. Neuroscience 30:741753.

King WM (1990) Nicotinic depolarization of optic nerve terminals augments synaptic transmission. Brain Res 527:150-154.

Lipton SA, Aizenman E, Loring RH (1987) Neural nicotinic acetylcholine responses in solitary mammalian retinal ganglion cells. Pfluegers Arch 410:37-43.

Luetje CW, Patrick J (1991) Both $\alpha$ - and $\beta$-subunits contribute to the agonist sensitivity of neuronal nicotinic acetylcholine receptors. $J$ Neurosci 11:837-845.

Manabe T, Renner P, Nicoll RA (1992) Postsynaptic contribution to potentiation revealed by the analysis of miniature synaptic currents. Nature 355:50-55.
Mulle C, Changeux J-P (1990) A novel type of nicotinic receptor in the rat central nervous system characterized by patch-clamp techniques. J Neurosci 10:169-175.

Mulle C, Vidal C, Benoit P, Changeux J-P (1991) Existence of different subtypes of nicotinic acetylcholine receptors in the rat habenulo-interpeduncular system. J Neurosci 11:2588-2597.

Mulle C, Choquet D, Korn H, Changeux J-P (1992) Calcium influx through nicotinic receptors in rat central neurons: its relevance to cellular regulation. Neuron $8: 135-143$.

Prusky GT, Shaw C, Cynader MS (1987) Nicotinic receptors are located on lateral geniculate terminals in cat visual cortex. Brain Res 412:131-138.

Rapier C, Lunt GG, Wonnacott S (1988) Stereo selective nicotineinduced release of dopamine from rat striatal synaptosomes: concentration dependence and repetitive stimulation. J Neurochem 50:11231130 .

Sahin M, Bowen WD, Donoghue JP (1992) Location of nicotinic and muscarinic cholinergic and $\mu$-opiate receptors in rat cerebral neocortex: evidence from thalamic and cortical lesions. Brain Res 579:135147.

Sargent PB (1993) The diversity of neuronal nicotinic acetylcholine receptors. Annu Rev Neurosci, in press.

Swanson LW, Simmons DM, Whiting PJ, Lindstrom J (1987) Immunocytochemical localization of neuronal nicotinic receptors in the rodent central nervous system. J Neurosci 7:3334-3342.

Vidal C, Changeux JP (1993) Nicotinic and muscarinic modulations of excitatory synaptic transmission in the rat prefrontal cortex in vitro. Neuroscience, in press.

Vernino S, Amador M, Luetje CW, Patrick J, Dani JA (1992) Calcium modulation and high calcium permeability of neuronal nicotinic acetylcholine receptors. Neuron 8:127-134.

Wada E, Wada K, Boulter J, Deneris E, Heinemann S, Patrick J, Swanson LW (1989) Distribution of $\alpha 2, \alpha 3, \alpha 4$ and $\beta 2$ neuronal nicotinic receptor subunit mRNAs in the central nervous system: a hybridization histochemical study in the rat. J Comp Neurol 284:314-335.

Wessler I (1992) Acetylcholine at motor nerves: storage, release, and presynaptic modulation (autoreceptors and adrenergic receptors). Int Rev Neurobiol 34:283-381.

Westfall TC (1974) Effect of nicotine and other drugs on the release of ${ }^{3} \mathrm{H}$-norepinephrine and ${ }^{14} \mathrm{C}$-dopamine in rat brain striatum and hypothalamus slices. Neuropharmacology 13:1025-1032.

Wonnacott S, Drasdo A, Sanderson E, Rowell P (1990) Presynaptic nicotinic receptors and the modulation of transmitter release. In: Ciba Foundation symposium, The biology of nicotine dependence, pp 87105. Chichester: Wiley.

Woolf NJ, Butcher LL (1985) Cholinergic systems in the rat brain. II. projections to the interpeduncular nucleus. Brain Res Bull 14:63-83. 